\title{
Improved detection of microbial ureteral stent colonisation by sonication
}

\author{
Gernot Bonkat $\cdot$ Malte Rieken $\cdot$ Cyrill A. Rentsch $\cdot$ Stephen Wyler • \\ Antje Feike · Juliane Schäfer - Thomas Gasser • Andrej Trampuz • \\ Alexander Bachmann • Andreas F. Widmer
}

Received: 26 December 2009/ Accepted: 5 March 2010/Published online: 21 March 2010

(C) Springer-Verlag 2010

\begin{abstract}
Purpose The diagnosis of microbial ureteral stent colonisation (MUSC) is difficult, since routine diagnostic techniques do not accurately detect microorganisms embedded in biofilms. New methods may improve diagnostic yield and understanding the pathophysiology of MUSC. The aim of the present study was to evaluate the potential of sonication in the detection of MUSC and to identify risk factors for device colonisation.

Methods Four hundred and eight polyurethane ureteral stents of 300 consecutive patients were prospectively evaluated. Conventional urine culture (CUC) was obtained prior to stent placement and device removal. Sonication was performed to dislodge adherent microorganisms. Data of patient sex and age, indwelling time and indication for stent placement were recorded.

Results Sonicate-fluid culture detected MUSC in 36\%. Ureteral stents inserted during urinary tract infection (UTI) were more frequently colonised (59\%) compared to those placed in sterile urine $(26 \% ; P<0.001)$. Female sex $(P<0.001)$ and continuous stenting $(P<0.005)$ were significant risk factors for MUSC; a similar trend was
\end{abstract}

G. Bonkat $(\bowtie) \cdot$ M. Rieken · C. A. Rentsch $\cdot$ S. Wyler .

A. Feike - T. Gasser - A. Bachmann

Department of Urology, University Hospital Basel,

Spitalstrasse 21, 4031 Basel, Switzerland

e-mail: bonkatg@uhbs.ch

\section{A. Trampuz · A. F. Widmer}

Division of Infectious Diseases and Hospital Epidemiology,

University Hospital Basel, Petersgraben 4,

4031 Basel, Switzerland

\section{J. Schäfer}

Institute for Clinical Epidemiology and Biostatistics, University

Hospital Basel, Hebelstrasse 10, 4031 Basel, Switzerland observed in patients older than 50 years $(P=0.16)$. MUSC and indwelling time were positively correlated $(P<0.005)$. MUSC was accompanied by positive CUC in $36 \%$. Most commonly isolated microorganisms were Coagulase-negative staphylococci $(18.3 \%)$, Enterococci (17.9\%) and Enterobacteriaceae (16.9\%).

Conclusions Sonication is a promising approach in the diagnosis of MUSC. Significant risk factors for MUSC are UTI at the time of stent insertion, female sex, continuous stenting and indwelling time. CUC is a poor predictor of MUSC. The clinical relevance of MUSC needs further evaluation to classify isolated microorganism properly as contaminants or pathogens.

Keywords Biofilm - Microbial colonisation - Sonication · Ureteral stent · Urinary tract infection

\section{Introduction}

Ureteral stents offer an ideal surface for microbial adherence and consecutive device colonisation. In the majority of cases, microbial ureteral stent colonisation (MUSC) remains asymptomatic. However, MUSC-related infectious complications are one of the leading serious risk factors associated with ureteral stent placement. During manipulation or instrumentation, biofilm organisms could be shed into the urine and lead to uncomplicated UTI up to urosepsis [1,2]. The issue of MUSC has not been well studied previously. Available data are inconsistent and controversial [2-10]. Microbiologic diagnosis of MUSC is difficult, since routine culture techniques do not accurately detect microorganisms embedded in biofilms. Sonication is a short, low-energy ultrasound treatment that may improve the detection of MUSC by liberating sessile biofilm 
organisms. However, most sonication devices have not been optimised for releasing pathogens from biofilms but killing pathogens susceptible to sonication. We developed a sonication system whose ultrasound power was monitored by a hydrophone and validated for optimal yield of bacteria and report the results of a prospective clinical trial using this new sonication technique for the detection of MUSC. The association between MUSC and CUC, UTI at the time of stent insertion, indwelling time, gender, single and continuous stenting as well as different indications for stent placement were investigated.

\section{Materials and methods}

Study population

All consecutive patients who had their stent removed were eligible for the study. The study has been approved by the local human subjects committee, and all included patients gave written informed consent.

\section{Laboratory investigations}

Ureteral stents were removed under aseptic conditions, divided into small parts, placed in sterile tubes and processed by the microbiology laboratory within $6 \mathrm{~h}$. Negative controls $(n=8)$ consisted of unused sterile ureteral stents unpacked in the operation room and sent for sonication to go through the same process as stents removed from the patients. CUC were obtained prior to stent insertion and removal. Stent colonisation was detected by sonication as described previously [11]. In brief, $10 \mathrm{ml}$ of Ringer's solution was added, tubes were vortexed for $30 \mathrm{~s}$ and sonicated (frequency, $40 \pm 2 \mathrm{kHz}$ and power density, $0.22 \pm 0.04 \mathrm{~W} / \mathrm{cm}^{2}$, as determined by a calibrated Hydrophone, Type 8103; Brüel and Kjær, Naerum, Denmark) in an Aquasonic Model 750T ultrasound bath (VWR Scientific Products) for $1 \mathrm{~min}$, followed by additional vortexing for $30 \mathrm{~s}$. The resulting sonicate fluid was plated in $0.5-\mathrm{ml}$ aliquots onto aerobic sheep blood agar plates (BD Diagnostic Systems) and incubated at $35-37^{\circ} \mathrm{C}$ in $5-7 \%$ carbon dioxide aerobically for 2 days. Microorganisms were enumerated and classified by routine microbiologic techniques.

\section{Definitions}

Single ureteral stenting

Stents obtained from patients undergoing singular stenting or the first stent removed from patients with continuous stenting.
Continuous ureteral stenting

Stents obtained from patients with continuous stenting with the exception of the first placed stent.

Positive sonicate-fluid culture (SFC)

Growth of $\geq 10^{2} \mathrm{CFU} / \mathrm{ml}$. Since no validated cut-off value for MUSC diagnosed by sonication exists, a threshold of $\geq 10^{2} \mathrm{CFU} / \mathrm{ml}$ was chosen according to recommendations for intravascular catheters [12].

Ureteral stent contamination

Growth of $<10^{2} \mathrm{CFU} / \mathrm{ml}$ and mixed gram-positive flora in SFC.

Positive urine culture (CUC) and urine contamination

CUC were interpreted according to Wilson et al. [13] regarding collecting technique, quantitation $(\mathrm{CFU} / \mathrm{ml})$ and number of microorganisms isolated.

Statistical analysis

Analyses were performed using R ( $\mathrm{R}$ Development Core Team 2009) [28] and the $R$ package geepack, which implements the generalised estimating equations (GEE) approach for fitting marginal generalised linear models to clustered data [29]. A $P$-value of less than 0.05 was considered to indicate statistical significance; all tests were two-sided. McNemar's chi-squared test and generalised estimating equations [30] were applied as appropriate.

\section{Results}

Four hundred and thirty polyurethane stents were removed during the study period. In patients with bilateral stents $(n=17)$, only one device was considered. Four patients refused consent, in one case CUC at the time of stent removal was not submitted to the laboratory. Data of 408 stents from 300 consecutive patients fulfilled the case definition.

Sonicate-fluid culture (SFC) detected MUSC in 36\%. Ninety-three discordant observational pairs were positive with sonication only, compared to eight positives with CUC only. All negative controls $(n=8)$ were negative in SFC.

UTI at the time of insertion offers a significant risk factor for MUSC (59.2\%) compared to sterile urine (26\%). The odds ratio, obtained from univariate GEE analysis assuming an exchangeable correlation structure, 
comparing patients with and without UTI at stent insertion was $3.16(95 \%$ confidence interval $(\mathrm{CI})=1.91-5.22$; $P<0.001)$.

The proportion of positive SFC was lower among devices obtained from singular stenting (30\%) compared to continuous stenting (49\%), whereas the odds ratio comparing continuous with single ureteral stenting was 1.96 (95\% CI $=1.25-3.08 ; P<0.005)$.

Stents obtained from female patients showed significantly higher positive SFC proportion (55\%) than stents obtained from males $(24 \%)$. The crude odds ratio comparing females with males was $3.72(95 \% \mathrm{CI}=2.35-5.89$; $P<0.001)$.

Duration of stenting and rate of MUSC were positively correlated. Stents left for up to 30 days, 31-90 days and more than 90 days were colonised in 27, 39 and 54\%, respectively (Table 1). The odds ratios comparing indwelling times of 31-90 days with $\leq 30$ days and $>90$ days with $\leq 30$ days were 1.67 (95\% $\mathrm{CI}=1.07-2.62$; $P=0.025)$ and 2.97 (95\% CI $=1.60-5.52 ; P=0.001)$. In order to avoid confounding, we included UTI at stent insertion, continuous stenting, gender, age and indwelling time as ordered categorical variables in a multivariable GEE analysis (Table 2).

Table 1 Stent characteristics

Data represent numbers, percents in parentheses, $S F C$ sonicate-fluid culture, UTI urinary tract infection
SFC encountered a total of 224 microorganisms (Table 3); i.e., on average 1.5 microorganisms per colonised stent. Thirty-three different organisms were identified and differentiated in nine subgroups. Coagulasenegative staphylococci spp. (CoNS) were most commonly isolated (18.3\%), followed by Enterococcus spp. (17.9\%) and Enterobacteriaceae (16.9\%). SFC observed single microbial growth in $59 \%$ and multiple growths in $41 \%$, respectively. From a total of 224 isolated microorganisms, $67 \%$ were gram-positive, while $22 \%$ were gram-negative and $11 \%$ fungi, respectively. Pure bacterial growth was found in $83 \%$, fungal in $9 \%$ and mixed growth in $8 \%$. The spectrum of microorganisms identified in cases with positive SFC and positive CUC correlated in $90 \%$. In $32 \%$, SFC revealed additional microbial growth. Most commonly isolated by CUC were Enterobacteriaceae 26\%, Enterococcus spp. $22 \%$ and Candida spp. 17\%. CUC missed MUSC in $64 \%$.

\section{Discussion}

Biofilm formation on ureteral stents was first noted in the early 1990s by Reid et al. [10]. Despite great interest in

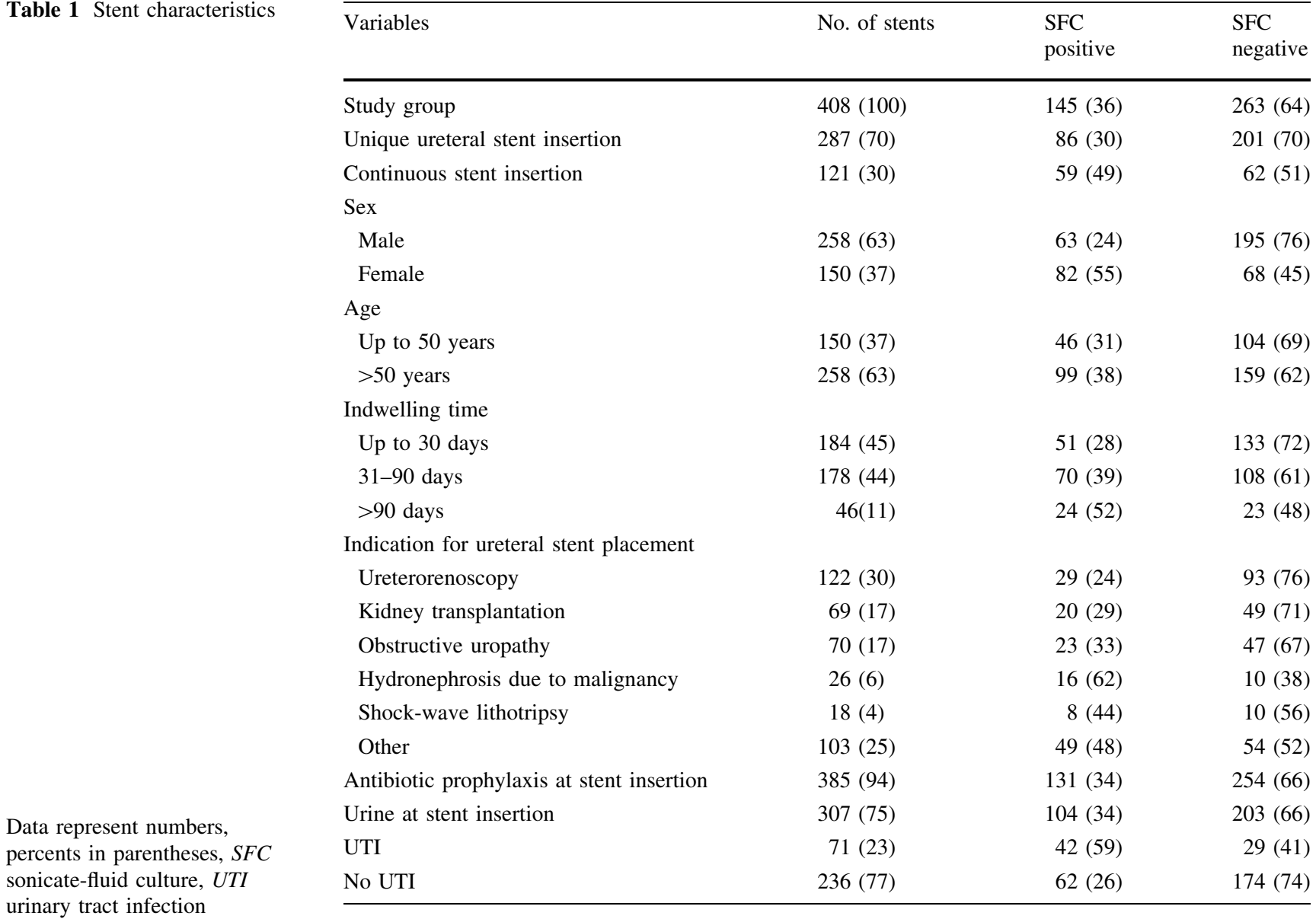


Table 2 Multivariable GEE fit of significant microbial stent colonisation using sonicate-fluid culture

\begin{tabular}{llllr}
\hline Covariate & Odds ratio & $95 \%$ CI & $P$-value \\
\hline UTI at stent insertion & 2.59 & 1.47 & 4.56 & 0.001 \\
Continuous stenting & 2.24 & 1.28 & 3.93 & 0.005 \\
Female sex & 3.35 & 1.89 & 5.94 & $<0.001$ \\
Indwelling time* $^{*}$ & & & & \\
Indwelling time $^{1}$ & 1.83 & 1.08 & 3.09 & 0.025 \\
Indwelling time $^{2}$ & 3.02 & 1.37 & 6.66 & 0.006 \\
Age $>50$ years $^{2}$ & 1.67 & 0.93 & 3.00 & 0.086 \\
\hline
\end{tabular}

* Indwelling time $\leq 30$ days as reference

${ }^{1}$ Indwelling time 31-90 days

${ }^{2}$ Indwelling time $>90$ days

Table 3 Microorganisms detected by sonicate-fluid culture

\begin{tabular}{|c|c|c|c|c|c|c|}
\hline Microorganism & Total no. & & Male sex & & Female sex & \\
\hline Jo. of microorganisms & 224 & $\mathrm{R}$ & 93 & $\mathrm{R}$ & 131 & \\
\hline $\mathrm{CoNS}^{1}$ & $41(18.3)$ & 1 & $25(26.9)$ & 1 & $16(12.2)$ & \\
\hline interococcus spp. & $40(1$ & 2 & 19( & 2 & 21 & \\
\hline Enterobacteriaceae $^{2}$ & 38 (16.9) & 3 & 12( & 3 & 26( & \\
\hline $\mathrm{Car}$ & 2 & 4 & 8) & 4 & 1 & \\
\hline Lactobacillus & 19( & 5 & 2( & 7 & 17 & \\
\hline Streptococcus $\mathrm{s}$ & $18(8$ & 6 & $6(t$ & 5 & $12(9.2)$ & \\
\hline Corynebacterium & $12(5.4)$ & 7 & $4(4.3)$ & 6 & $8(6.1)$ & \\
\hline Gardner & $6(2.7)$ & 8 & $1(1.1)$ & 8 & $5(3.8)$ & \\
\hline Other $^{3}$ & $26(11.6)$ & 9 & $13(14)$ & 9 & $13(9.9)$ & \\
\hline
\end{tabular}

$\mathrm{R}$ (rank), data represent numbers, percents in parentheses

${ }^{1}$ Coagulase-negative staphylococci spp

${ }^{2}$ Escherichia coli $(n=27)$, Serratia marcescens $(n=2)$, Citrobacter freundii $(n=1)$, Proteus mirabilis/peneri $(n=3)$, Klebsiella pneumoniae $(n=2)$, Enterobacter cloacae $(n=1)$, Enterobacter aerogenes $(n=1)$

${ }^{3}$ Kocuria rosea $(n=5)$, Kocuriae kristinae $(n=3)$, Dermacoccus nishinomiyaensis $(n=1)$, Staphylococcus aureus $(n=8)$, Pseudomonas aeruginosa $(n=4)$, Erysipelothrix rhusiopathiae $(n=1)$, Actinobaculum Schaalii $(n=1)$, Moraxella catarrhalis $(n=1)$, Gram-labile rods $(n=2)$

clinical-related biofilm research to date, only a few studies conducted further investigations in this field [2-10]. Available data about MUSC are inconsistent and controversial.

In the majority of previous studies, a detailed description regarding the microbiological diagnostic technique is missing. Kehinde et al. [8] used the semiquantitative role and plate method and observed MUSC in $42 \%$. Klis et al. [9] reported about MUSC in $98.5 \%$ detected by qualitative broth culture. However, these two diagnostic techniques have limitations like the risk of contamination [14]. Furthermore, qualitative broth culture cannot quantify the number of microorganisms; thus, it is not possible to differentiate between MUSC and contamination. A further disadvantage of the role and plate method is that it allows detection of outer surface bacteria only.

Sonication is a short, low-energy ultrasound treatment that improves the diagnosis of microbial medical device colonisation by liberating organisms encased in biofilms [11, 15-17]. Technically, ultrasound waves radiate through a solution and produce high- and low-pressure areas. During the low-pressure stage, millions of microscopic vapour bubbles are formed, which collapse during the highpressure stage releasing an enormous amount of energy on the surface of the objects. This agitation causes a vacuumscrubbing action by releasing acoustic energy at the surface of objects [18, 19]. We predominantly detected high numbers of organisms (at least $10^{2} \mathrm{CFU}$ per plate). Quantification of the number of microorganisms and detection of polymicrobial growth is another advantage of sonication. Although trials have evaluated the potential of sonication in diagnosing MUSC previously [4, 7, 10], the results of these studies have to be taken with precaution, since they bear methodological flaws. Ultrasound is defined by frequencies above the range of human hearing $(20 \mathrm{kHz})$. Two studies [4, 7] used frequencies of $50 \mathrm{~Hz}$, and in one study, technical details of sonication were missing [10]. In addition, biofilm removal by sonication strongly depends on the intensity of sonication energy (power density) and to a far lesser extent on frequency [20]. Inappropriate power density may lead to elimination of microorganisms due to thinning of cell membranes, localised heating and production of free radicals [21]. Gram-negative bacteria were more susceptible to these detrimental effects than grampositives due to the lack of a thick and robust cell wall. The relative high proportion of gram-positive microorganisms detected in previous studies using sonication $[4,7,10]$ may be attributed to the elimination of gram-negative bacteria by ultrasound due to inappropriate power densities. Therefore, measuring power density by a calibrated hydrophone should be an obligatory step prior to using sonication. A disadvantage of sonication is the potential release of biofilm organism that might not be clinically relevant.

The role of CUC in predicting MUSC was discussed previously [2-8]. However, an exact differentiation between pathogens and contaminating organisms is missing in all studies, resulting in a higher rate of positive CUC when compared to our results. We could confirm the reported impaired value of CUC in predicting MUSC. However, this is not astonishing since CUC can only detect free floating. Positive CUC was missing in about $60 \%$ of colonised stents. Since MUSC does not necessarily lead to bacteriuria, negative CUC does not rule out stent colonisation. The higher prevalence of MUSC on devices 
obtained from continuous stenting compared to single stenting is explainable with the replacement technique via a guide wire. The wire may displace intraluminal biofilm fragments, and the new device could be inoculated as it slides over. The rate of MUSC was significantly higher in stents removed from females compared to those from males. Since UTI and bacteriuria are more common in women than in men, this observation is comprehensible. Previous studies showed similar results [2-4, 8]. The increase in MUSC in stents derived from patients older than 50 years might be associated with the age-related increase in bacteriuria. In accordance with previous studies $[2,3,7]$, we observed a positive correlation between indwelling time and rate of MUSC.

We predominantly detected CoNS, Enterococci spp. and Enterobactericeae by SFC. The variation of isolated microorganisms compared to other studies may be explainable with variations in the spectrum of different hospitals and countries [8], the diagnostic technique and the study population. CoNS have long been dismissed as culture contaminants. Today, they are recognised as one of the most frequent causes of biofilm-associated infections $[22,23]$. CoNS frequently colonise skin and mucus surfaces. Any medical device that penetrates those surfaces during surgery is at high risk to become colonised. The high prevalence of CoNS in the distal male urethra [24] may be responsible for the frequent detection of these organisms on stents obtained from male patients. Enterococcus spp. have been associated with biofilms on various kinds of indwelling medical devices [25]. In the present trial, Escherichia coli was the most common gram-negative organism isolated by SFC. This gram-negative rod uses several virulence factors like fimbrial protein fimH. Fimbrial protein binds to Tamm-Horsfall protein (THP). THP has been found to adhere to ureteral stents [26] and therefore may act as a promoter of MUSC [27].

How could our findings be transferred into clinical practice? Although during stent manipulation or instrumentation, undetected biofilm organisms may be shed into the urine, serious infectious complications [1, 2] are rare. Thus, we could not recommend sonication as a standard procedure in every patient. However, once a serious biofilm-associated infectious complication occurs, the attending clinician might be surprised by his microbiological opponents. Microbiological investigations like blood and urine cultures would be initiated. But the quantity, aggressiveness and susceptibility pattern of the responsible pathogen remain unknown for at least 24-48 h and initial antimicrobial treatment is empiric. Patients especially with a high risk of infectious complications (e.g. patients with immunosuppressants, diabetes mellitus or artificial cardiac valve) would benefit from an early identification of biofilm organisms by sonication.
Reid et al. [10] posed the question how important is MUSC in asymptomatic patients? We would add the question, how important is MUSC in symptomatic patients? Is it possible that patients with an indwelling ureteral stent develop symptoms due to MUSC? A prospective clinical trial analysing the relationship between MUSC, LUTS and other stent-related symptoms could elucidate this issue. Moreover, these data might improve pharmaceutical treatment and the development of appropriate coatings to delay stent colonisation.

Some limitations of this study should be mentioned: (1) The current lack of a gold standard definition of MUSCrelated infection (2) No validated microcobiological gold standard technique exist for the diagnosis of MUSC (3) The cut-off value of SFC for MUSC was chosen according to a reference for intravascular catheters (4) Only conventional microbiologic culture techniques were applied to identify microbial growth. Novel and more sensitive approaches like immunfluorescence microscopy as well as amplification of the $16 \mathrm{~S}$ rRNA may be more sensitive to detect fastidious organisms.

\section{Conclusion}

The results of our study demonstrate that sonication is a promising approach in the diagnosis of MUSC. Results of CUC are a poor predictor of MUSC. Stents placed during UTI and devices derived from females, patients older than 50 years and continuous stenting are at major risk to be colonised. The clinical relevance of MUSC needs further evaluation to classify isolated microorganism properly as contaminants or pathogens.

Conflict of interest statement There is no conflict of interest.

\section{References}

1. Gautam G, Singh AK, Kumar R, Hemal AK, Kothari A (2006) Beware! Fungal urosepsis may follow endoscopic intervention for prolonged indwelling ureteral stent. J Endourol 20(7):522524

2. Riedl CR, Plas E, Hubner WA, Zimmerl H, Ulrich W, Pfluger H (1999) Bacterial colonization of ureteral stents. Eur Urol 36(1):53-59

3. Farsi HM, Mosli HA, Al-Zemaity MF, Bahnassy AA, Alvarez M (1995) Bacteriuria and colonization of double-pigtail ureteral stents: long-term experience with 237 patients. J Endourol 9(6):469-472

4. Keane PF, Bonner MC, Johnston SR, Zafar A, Gorman SP (1994) Characterization of biofilm and encrustation on ureteric stents in vivo. Br J Urol 73(6):687-691

5. Lifshitz DA, Winkler HZ, Gross M, Sulkes J, Baniel J, Livne PM (1999) Predictive value of urinary cultures in assessment of 
microbial colonization of ureteral stents. J Endourol 13(10):735738

6. Lojanapiwat B (2006) Colonization of internal ureteral stent and bacteriuria. World J Urol 24(6):681-683

7. Paick SH, Park HK, Oh SJ, Kim HH (2003) Characteristics of bacterial colonization and urinary tract infection after indwelling of double-J ureteral stent. Urology 62(2):214-217

8. Kehinde EO, Rotimi VO, Al-Hunayan A, bdul-Halim H, Boland F, Al-Awadi KA (2004) Bacteriology of urinary tract infection associated with indwelling $\mathrm{J}$ ureteral stents. $\mathrm{J}$ Endourol 18(9):891-896

9. Klis R, Korczak-Kozakiewicz E, Denys A, Sosnowski M, Rozanski W (2009) Relationship between urinary tract infection and self-retaining double-J catheter colonization. J Endourol 23(6):1015-1019

10. Reid G, Denstedt JD, Kang YS, Lam D, Nause C (1992) Microbial adhesion and biofilm formation on ureteral stents in vitro and in vivo. J Urol 148(5):1592-1594

11. Trampuz A, Piper KE, Jacobson MJ et al (2007) Sonication of removed hip and knee prostheses for diagnosis of infection. N Engl J Med 357(7):654-663

12. Mermel LA, Allon M, Bouza E et al (2009) Clinical practice guidelines for the diagnosis and management of intravascular catheter-related infection: 2009 update by the infectious diseases society of America. Clin Infect Dis 49(1):1-45

13. Wilson ML, Gaido L (2004) Laboratory diagnosis of urinary tract infections in adult patients. Clin Infect Dis 38(8):1150-1158

14. Costerton W, Veeh R, Shirtliff M, Pasmore M, Post C, Ehrlich G (2003) The application of biofilm science to the study and control of chronic bacterial infections. J Clin Invest 112(10):1466-1477

15. Padberg FT Jr, Smith SM, Eng RH (1992) Optimal method for culturing vascular prosthetic grafts. J Surg Res 53(4):384-390

16. Tunney MM, Patrick S, Gorman SP et al (1998) Improved detection of infection in hip replacements. A currently underestimated problem. J Bone Joint Surg Br 80(4):568-572

17. Sherertz RJ, Raad II, Belani A et al (1990) Three-year experience with sonicated vascular catheter cultures in a clinical microbiology laboratory. J Clin Microbiol 28(1):76-82

18. Pitt WG, Ross SA (2003) Ultrasound increases the rate of bacterial cell growth. Biotechnol Prog 19(3):1038-1044
19. Trampuz A, Osmon DR, Hanssen AD, Steckelberg JM, Patel R (2003) Molecular and antibiofilm approaches to prosthetic joint infection. Clin Orthop Relat Res 414:69-88

20. Pitt WG (2005) Removal of oral biofilm by sonic phenomena. Am J Dent 18(5):345-352

21. Piyasena P, Mohareb E, McKellar RC (2003) Inactivation of microbes using ultrasound: a review. Int $\mathrm{J}$ Food Microbiol 87(3):207-216

22. von Eiff EC, Peters G, Heilmann C (2002) Pathogenesis of infections due to coagulase-negative staphylococci. Lancet Infect Dis 2(11):677-685

23. Diekema DJ, Pfaller MA, Schmitz FJ et al (2001) Survey of infections due to Staphylococcus species: frequency of occurrence and antimicrobial susceptibility of isolates collected in the United States, Canada, Latin America, Europe, and the Western Pacific region for the SENTRY antimicrobial surveillance program, 1997-1999. Clin Infect Dis 32(Suppl 2):S114-S132

24. Montagnini SD, Mamizuka EM, Pereira CA, Srougi M (2000) Microbiologic aerobic studies on normal male urethra. Urology 56(2):207-210

25. Donlan RM, Costerton JW (2002) Biofilms: survival mechanisms of clinically relevant microorganisms. Clin Microbiol Rev 15(2):167-193

26. Santin M, Motta A, Denyer SP, Cannas M (1999) Effect of the urine conditioning film on ureteral stent encrustation and characterization of its protein composition. Biomaterials 20(13):1245-1251

27. Chew BH, Lange D (2009) Ureteral stent symptoms and associated infections: a biomaterials perspective. Nat Rev Urol 6(8):440-448

28. R Development Core Team (2009) R: a language and environment for statistical computing. $\mathrm{R}$ foundation for statistical computing, Vienna, Austria, ISBN 3-900051-07-0. http://www. R-project.org/

29. Halekoh U, Højsgaard S, Yan J (2006) The R package geepack for generalized estimating equations. J Statist Soft 15(2):1-11. http://www.jstatsoft.org/v15/i02/

30. Liang K-Y, Zeger SL (1986) Longitudinal data analysis using generalized linear models. Biometrika 73(1):13-22 